\title{
Nano-Engineering of Graphene and Related Materials
}

\author{
Zhiping $\mathrm{Xu}$ \\ Tsinghua University \\ China
}

\section{Introduction}

Graphene is the ultimately monolayer material with single-atom thickness. It possesses many fascinating properties including massless Dirac electronic structure, anomalous quantum Hall effects, high mobility, extraordinary high thermal conductivity, stiffness and strength (see other chapters in this book). Impressively, these properties have remarkable dependence on its morphology and atomic structure, e.g. its size and shape (Fig. 1, Xu \& Buehler, 2010a). Moreover, interior and exterior doping effects from defects, molecular adsorptions or electromagnetic fields are also significant. For example, hydrogenation, oxidation and electrical field significantly tune its structural, mechanical, thermal, electronic and optical structures, which is controllable and even reversible. These sensitive responses of a graphene sheet to structural and environmental cues enable us opportunities to engineer its physical and chemical properties at nanoscale.

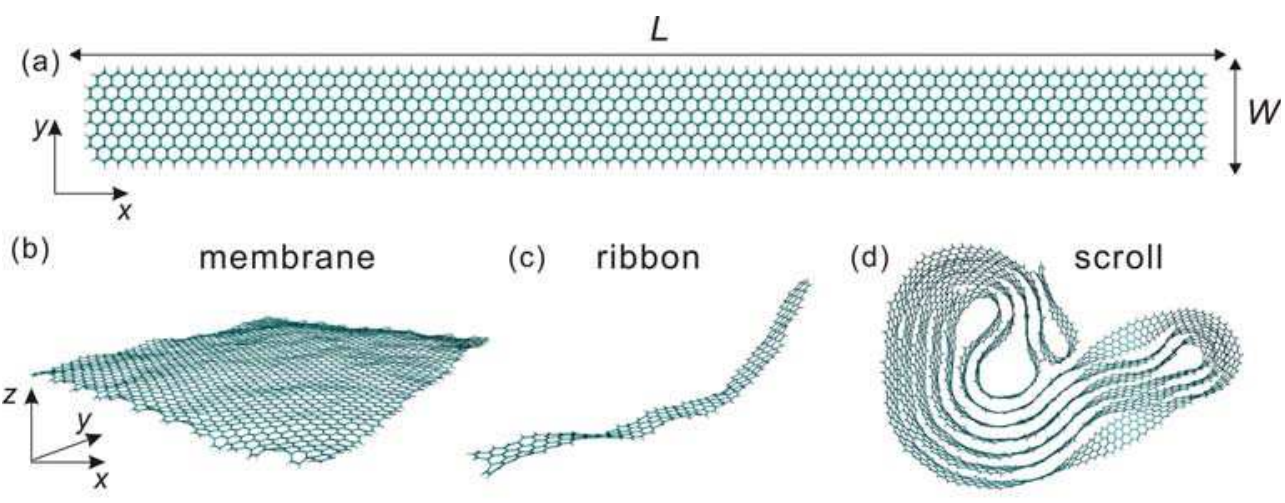

Fig. 1. A phases diagram for graphene sheets as a function of their geometric parameters: (a) a graphene sheet with length $L$ and width $W$; (b) a two-dimensional membrane with ripples from thermal fluctuation as $L \sim W$; (c) a fluctuating one-dimensional ribbon while it has apparent aspect ratio $n=L / W$; (d) a nanoscroll after a ribbon self-folds due to the van der Waals interactions between adjacent graphene sheet. (Xu \& Buehler, 2010a) 


\section{Nano-engineering by tailoring nanostructures}

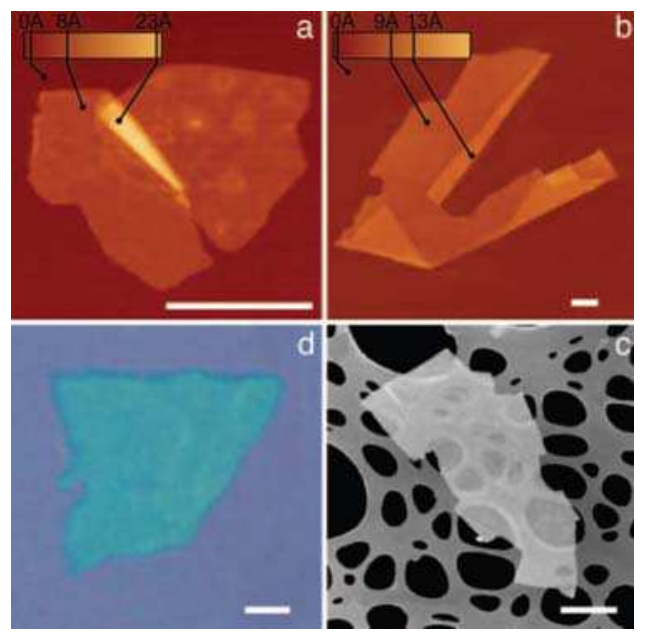

Fig. 2. Two-dimensional monolayer crystallites of $\mathrm{NbSe}_{2}(\mathrm{a})$, graphite (b), $\mathrm{Bi}_{2} \mathrm{Sr}_{2} \mathrm{CaCu}_{2} \mathrm{O}_{x}$ (c) and $\mathrm{MoS}_{2}(\mathrm{~d})$ as prepared by the mechanical exfoliation technique and visualized by atom force microscopy (AFM) (a and b), scanning electron microscopy (c) and optical microscope (d). (All scale bars: one micrometer) (Novoselov et al., 2005)

Back to year 2004, the first experimental establishment of producing graphene sheets is achieved by Andre Geim and Konstantin Novoselov of the University of Manchester, through a so-called "scotch-tape" technique (Novoselov et al., 2004). In this approach, single- or few-layered graphene sheets can be obtained by repeatedly peeling highly oriented pyrolytic graphite (HOPG) using scotch tapes. Although the graphene sheets thus produced are poor in the production yield and controllability on the geometry, this mechanical exfoliation technique features high reliability and offers graphene sheets of very high quality. Even at present, the simple but efficient "scotch-tape" technique is still a common one to fabricate high-quality samples. This seminal work is our first time to fabricate and observe two-dimensional crystalline materials. Geim and Novoselov are thus awarded the 2010 Nobel Prize in physics. In prior to their work, physicists believed that two-dimensional crystals couldn't sustain stabilities in ambient environment at finite temperature. Early work based on elasticity theory shows that the out-of-plane displacement field diverges for infinite large two-dimensional crystals (Mermin \& Wagner, 1966). However, the observation of stable graphene, $\mathrm{BN}, \mathrm{MoS}_{2}$ and other monolayers (Fig. 2) gives rise to a the contradiction to the theoretical prediction. It is realized later that the monatomic layers are stablized by the anharmonic coupling between bending and stretching modes in the graphene sheet, i.e. forming out-of-plane ripples and wrinkles on the graphene sheet maintains its planar morphology (Fig. 1(b)) and avoids collapsing into folds and scrolls (Fig. 1(d)). The morphology of graphene sheet are thus determined by the size, shape, temperature and boundary conditions. Recent atomistic simulations (Fasolino, Los \& Katsnelson, 2007; Xu \& Buehler, 2010a) and experiments (Meyer et al., 2007) clarify the underlying mechanism and predict the morphology phase diagram for graphene. 
Inspired by this exciting discovery and novel physics uncovered in subsequent experiments, numerous efforts have been made to fabricate graphene sheets of high quality, and low price in parallel. In addition to mechanical exfoliation technique that produces micron-sized graphene flakes, chemical vapour deposition (CVD) on metal surface has also been successfully applied to synthesize single- and few-layer graphene sheet with areas of square centimetres (Bae et al., 2010). However, the most popular method for mass production at present is the chemical reduction technique, where graphite is firstly exfoliated in an oxygenrich environment. The intermediate product, graphene oxide, is subsequently reduced to recover bare graphene sheet. In addition, there are also several novel approaches available now, such as cutting single-walled carbon nanotubes (Kosynkin et al., 2009; Jiao et al., 2009) and bottom-up assembling from organic molecules (Yang et al., 2008; Cai et al., 2010).

The bulk graphene sheet is a semi-metal with a linear dispersion around the Dirac point. However when one of the in-plane dimensions reduces below $20 \mathrm{~nm}$ while maintaining the other dimension, the graphene ribbon (Fig. 1(c)) will feature an edge-dependent electronic structure due to the quantum confinement and lattice symmetry (Son, Cohen \& Louie, 2006a and 2006b). Armchair-edged graphene nanoribbons (AGNR) have width-dependent energy gaps, while a zigzag-edged nanoribbons (ZGNR) is always metallic with a localized state at the edge when the spin is unpolarized. This unique structure-property relationship suggests that simple geometrical tailoring could realize effective modification on the properties of graphene.

\subsection{Graphene nanostructures as building blocks for nanoelectronics}

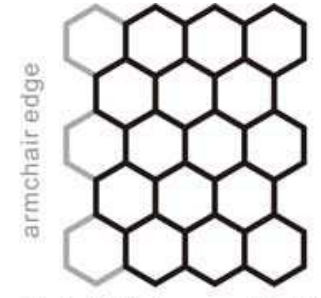

$N_{\mathrm{a}} 12234$

$N-1 N$ (a)

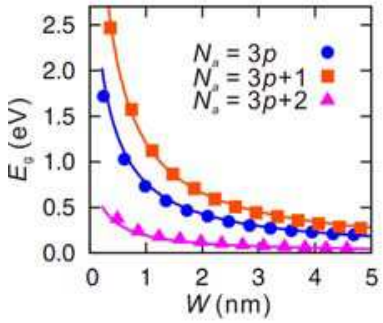

(c)

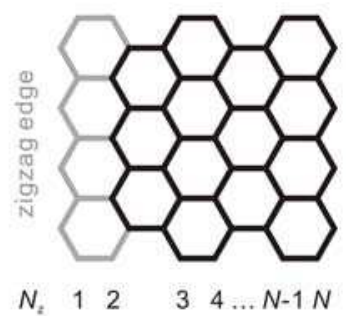

(b)

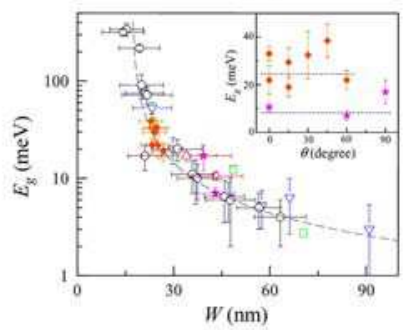

(d)

Fig. 3. (a) and (b) Atomic structures of armchair and zigzag GNRs; (c) Bandgap of armchair graphene nanoribbons calculated from density functional theory (Son, Cohen \& Louie, 2006); (d) Experimental measured bandgaps of graphene nanoribbons and their dependence on the orientation of crystalline lattice (Han et al., 2007). 
As illustrated in Fig. 3, the width of AGNR structure can be denoted by the number $N_{a}$. Tight-binding analysis shows that (Ezawa, 2006; Son, Cohen \& Louie, 2006a and 2006b) when $N_{a}$ is $3 p+2$ (where $p$ is a positive integer), the AGNR is metallic. While when $N_{a}$ is $3 p$ or $3 p+1$, the bandgap of AGNR is inversely proportional to its width $\left(E_{\mathrm{g}(3 p)} \geq E_{\mathrm{g}(3 p+1)}\right)$. These results are consistent with density functional theory (DFT) calculations based on local density approximation (LDA). The only difference is that in the DFT simulation, the reconstruction of structure and electron density at ribbon edge opens small gap for $N_{a}=$ $3 p+2$, and decreases (increases) the gaps of $3 p(3 p+1)$ AGNRs.

The structure of ZGNR is shown in Fig. 3(b). Simple analysis from the tight-binding picture predicts that the spin-unpolarized eigenstates of ZGNRs near $E_{\mathrm{F}}$ have a peculiar edge-state structure. There are two edge states decaying into the center of ZGNRs with a profile depending on their momentum. This can be seen from the flat bands in Fig. 3 for $k>2 \Pi / 3$, which give rise to a very large density of states at $E_{\mathrm{F}}$ and infinitesimally small on-site repulsion, could make the ZGNRs magnetic (Fujita et al., 1996; Nakada et al., 1996). Son, Cohen and Louie further find that when a transverse electric field is applied, an energy gap is opened for one spin channel while the other remains metallic (Son, Cohen \& Louie, 2006a and 2006b).

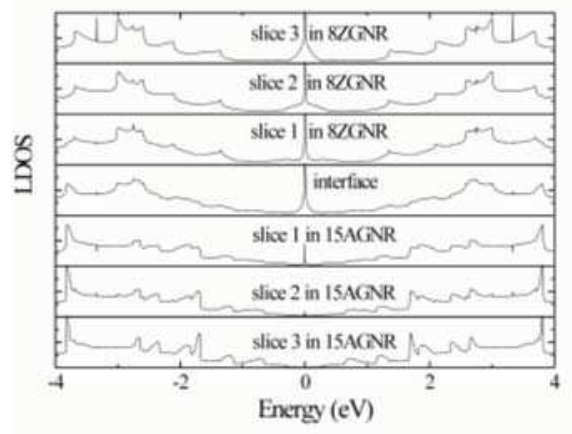

(a)
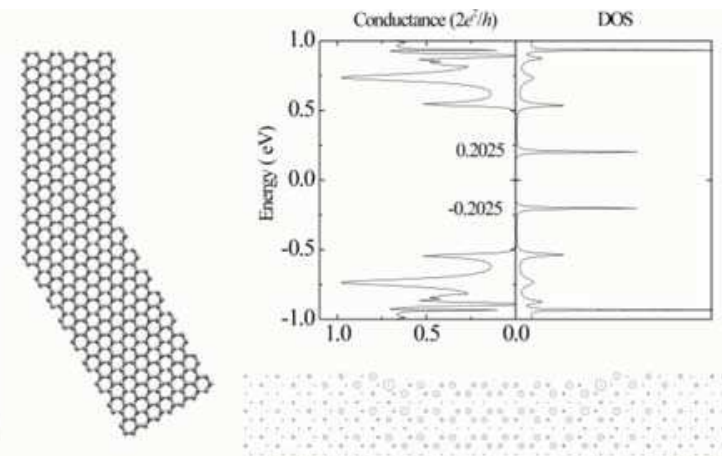

(b)

Fig. 4. GNR junctions based building blocks for nanoelectronics: (a) a junction connecting metallic ZGNRs and semiconducting AGNRs form a Schottky barrier at the interface; (b) a sandwich AGNR structure with varying width behaves as a quantum dot, the energy levels are tunable through varying the width of the central GNR (Xu, Zheng \& Chen, 2007).

The width-dependence energy gaps in AGNR are soon verified by experiments (Chen et al., 2007; Han et al., 2007). Interestingly, Han et al. also find that the bandgap $E_{\mathrm{g}}$ shows no direct dependence on the crystallographic direction (Han et al., 2007). Actually during the fabrication processes or during the self-passivating process after the structure is formed, the edge shape (armchair or zigzag) can reconstruct (Jia et al., 2009; Girit et al., 2009) and this may cause the bandgap to appear for graphene nanoribbons with any crystalline orientation.

As mentioned above, graphene nanoribbons feature remarkable size- and edge-dependent electronic properties. Elementary building blocks for nanoelectronics, such as metal/semiconductor junctions and quantum dots, thus can be constructed through a combination of these structural patterns (Xu, Zheng \& Chen, 2007). As shown in Fig. 4(a), a junction combining metallic and semiconducting GNRs is easy to form. By design a 
sandwich structure as shown in Fig. 4 (b), a quantum dots with tunable energy levels can also be achieved.

Moreover, Finite-sized graphene nanodisk is another graphene derivative with close edges. They feature similar electronic structure as the GNRs. The triangular zigzag nanodisk can be interpreted as a quantum dot with an internal degree of freedom. Ezawa find that its ground state is a quasi-ferromagnet, which is a ferromagnetic-like state with a finite but very long lifetime. A combined system including nanodisks and leads can find applications in spin filters, amplifiers and diodes (Ezawa, 2009). Kaxiras's group also investigate arbitrarily shaped graphene nanoflakes with focuses on the spin properties and propose spintronic nanoscale devices by sculpting graphene fragments and exploiting the shape dependence of magnetic properties (Wang et al., 2007). Other nanostructures of graphene such as twists (Bets \& Yakobson, 2009; Gunlycke et al., 2010) and scrolls (Xie et al., 2009; Shi et al., 2010) also have interesting electronic properties and potential applications.

\subsection{Synthesis and fabrication of graphene nanostructures}

To achieve a rational design of graphene nanostructures as suggested in Section 2.1, experimental techniques such as catalytic hydrogenation based nanocutting method are proposed to fabricate graphene nanostructures with specific geometry.

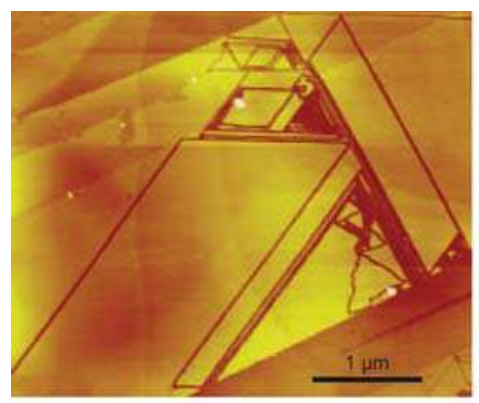

(a)

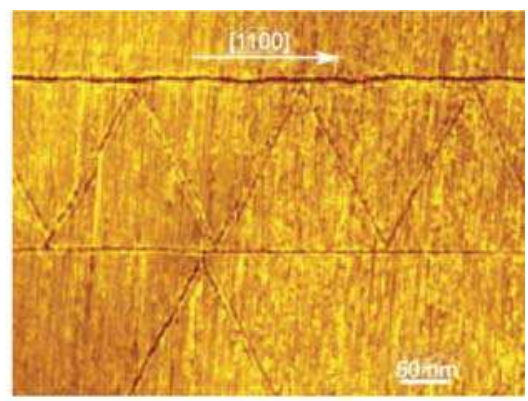

(b)

Fig. 5. Graphene nanostructures fabricated by catalytic hydrogenation: (a) an AFM images of graphene nanoribbons with different sizes and shapes; (b) STM image of equilateral triangle shaped graphene flakes formed by channels along [1-100] orientation (Ci et al., 2008).

Ci et al. (Ci et al., 2008) report a controlled cutting process for graphene sheets, using nickel nanoparticles as a knife that cuts with nanoscale precision (Fig. 5). The cutting proceeds via catalytic hydrogenation of the graphene lattice, where carbon atoms in graphene react with hydrogen into hydrocarbon and the catalytic nanoparticles continue to move due to the adhesion from remainder carbon atoms in graphene. This cutting process generates graphene pieces with specified zigzag or armchair edges. The size of the nanoparticle dictates the width of etched channel and edge structure that is produced during the cutting. The cutting occurs along straight lines and along symmetry lines, defined by angles of $60^{\circ}$ or $120^{\circ}$, and is deflected at free edges or defects, allowing practical control of graphene nanoengineering. DFT calculation shows that the reaction barrier to remove carbon atoms and leave zigzag edges is much higher than armchair edges. This explains the experimental observation where zigzag edges are dominant. 
Kinetic Monte Carlo method based on DFT results is applied to simulate the cutting dynamics. The result indicates that the nickel nanoparticle prefers to keep a straight trajectory. However when it encounters a void or open edge, the cohesion between carbon and nickel atoms force it to turn or retract back into the graphene. Thus with predefined voids or channels, specific cutting patterns can be created. Example nanostructures as fabricated using this technique, such as triangular and rectangular graphene sheets, are shown in Fig. 5.

Scanning tunnelling microscope lithography is also used to patterning graphene sheets into nanoribbons, bent junctions with nanometer-precision, well-defined widths and predetermined crystallographic orientations (Tapasztó et al., 2008). Furthermore, oxidation induced epoxy group can assist the lithography further (Fujii \& Enoki, 2010).

Back to the "scotch tape" approach, as the armchair edge has lower energy than the zigzag edge, thus tearing the graphene sheet along either armchair or zigzag direction will be selfdirected to leave armchair edges (Kawai et al., 2009). If the graphene sheet is attached to an adhesive substrate, the peeling process and edge geometry are then determined by both the tearing direction and substrate cohesion (Sen et al., 2010).

It is observed that the nanostructures thus fabricated have their confinement gaps opened up to $0.5 \mathrm{eV}$, enabling room-temperature operation of graphene nanoribbon-based devices. The methods introduced above are all top-down approaches. These methods avoid the difficulties of assembling nanoscale components and may prove useful in the realization of complete integrated circuits, operating as room-temperature ballistic electronic devices. However, recent progresses also show that chemical methods that based on self-assembly reaction of monomers are also feasible. Using building blocks such as 10,10'-dibromo-9, $9^{\prime}$ bianthryl, graphene nanoribbons with atomic precision can also be synthesized (Yang et al., 2008; Cai et al., 2010).

\section{Molecular doping on the graphene sheets}

In comparison to three-dimensional bulk semiconductors such as silicon, the two-dimensional morphology and single-atom thickness offer the graphene sheet more activity flexibility in response to either interior defects or exteriorly physical or chemical adsorptions. In graphene, each carbon atom is exposed to the environment with a dangling $\Pi$ bond, and thus is amiable to molecular functionalization on the basal plane. It is remarkable that the functionalization process could be reversed by breaking the bonding between adsorptions and graphene sheet. For example, in the chemical reduction process for producing graphene sheets, graphene monolayers are firstly exfoliated from graphite by oxidation, where the epoxy and hydroxyl binding on the basal plane of graphene break the interlayer binding. The oxidation groups are subsequently reduced to recover graphene sheets. Recently there are arising efforts in engineering the physical chemistry of graphene by functionalization, especially in the extent of molecular doping, covalently or non-covalently. In addition to the approaches in bulk materials such as substitutions, vacancies and interstitials, the notion of doping has been renovated here in a controllable and even reversible manner thanks to the coexistence of chemical inertness of $s p^{2}$ carbon network and relative activity of the $p_{z}$ electrons therein.

\subsection{Molecular doping on the basal plane of graphene sheets}

Non-covalent functionalization, or physisorption refers to the process in which the electronic structure of graphene is barely perturbed. The weak binding nature can be established through forces such as van der Waals or electrostatic dipole-dipole interactions. 
These functionalizations, especially those are weak in mechanical binding but strong in electronic coupling, hold great promises in the various applications such as chemical sensors, molecular switches and transistors.

The fundamental limit for these applications is the fluctuations due to thermal motion of charges and defects that lead to intrinsic noise exceeding the responsive signal from individual adsorption molecules. Impressively, gaseous non-covalent dopants, such as the water molecule, $\mathrm{N}_{2}, \mathrm{NO}_{2}$ and $\mathrm{NH}_{3}$, can be adsorbed physically on the basal plane of graphene. These adsorptions, although interacting weakly with graphene, have remarkable impacts on the intrinsic electronic properties of graphene. The Hall measurements show that $\mathrm{NO}_{2}, \mathrm{H}_{2} \mathrm{O}$ and iodine act as acceptors whereas $\mathrm{NH}_{3}, \mathrm{CO}$ and ethanol are donors. They contribute to a resistivity change up to 4 percent at zero magnetic field (Schedin et al., 2007). The adsorbed molecules change the local carrier concentration in graphene one by one electron, and lead to step-like changes in resistance. The micro-sized graphene thus can detect individual events when a gas molecule attaches to or detaches from its surface. Chemical sensors and nonvolatile memories can be directly designed following this concept.

Similarly, the non-covalent binding of 2,3-dichloro-5,6-dicyano-1,4-benzoquinone (DDQ) and tetrathiafulvalene (TTF) on graphene sheet induce the hybridization between the molecular levels and graphene valence bands, and transform the zero-gap semiconducting graphene into a metallic graphene. However, The functionalizations by non-covalent organic molecules physisorptions cannot lead to significant response in the transport property for sensory applications. Instead, by introducing metal atoms to intercalate between the molecules and graphene sheet, a two-order higher response can be achieved (Zhang et al., 2009).

Not only non-covalent adsorptions, but also some chemisorptions that forming covalent bonds with carbon atoms on the basal plane of graphene can tune its properties in a controllable manner. Hydrogenation and oxidation are two of the most noticeable approaches in the covalent context. Electrochemical modification of graphene can be induced by chemical reactions of graphene with proton and hydroxyl that are catalytically generated from water molecules in the gate dielectrics, making the graphene nonconductive. Notably, this process can subsequently be reversed by short current pulses that cause rapid local annealing (Echtermeyer et al., 2007). Experimental studies also reveal the semimetalinsulator transition in graphene after hydrogenation, which is then recoverable through structural annealing (Elias et al., 2009). When rationally designed, this reversible engineering on graphene has further potential applications in designing functional nanoscale materials and devices, such as hydrogen storage materials and patterned nanoelectronics (Singh \& Yakobson, 2009; Lee \& Grossman, 2010). The fully hydrogenated graphene - graphane - offers a high hydrogen storage weight ratio up to $7.7 \mathrm{wt} \%$ that is able to meet US Department of Energy (DOE)'s 2010 goal (6 wt \%).

Oxidation of graphene sheet has been widely utilized in fabricating graphene sheet in the chemical reduction method to exfoliate monatomic layer from graphite. The most notable structure for the intermediate product, graphene oxide, proposed is the Lerf-Klinowski model on the basis of NMR spectroscopy data, which contains epoxy and hydroxyl groups on the graphene sheets or at their edges (Lerf et al., 1998). Whereas hydroxyl groups are easy to be removed under annealing, the epoxy groups are much more stable. Experimentally, fault lines in graphene are observed experimentally in oxidized graphene, where epoxy group unzip the $s p^{2}$ carbon-carbon bonds beneath ( $\mathrm{Li}$ et al., 2006). This is explained as adsorbed epoxy groups that tend to line up cooperatively for lower formation 
energy. In contrast, a recent ultrahigh vacuum scanning probe microscopy (UHV STM) study reveals locally periodic structures where the hexagonal lattice of graphene is only distorted slightly. The atomic structure is identified as an oxygen atom lattice bound to the graphene sheet with a high density of $n_{\mathrm{O}}: n_{\mathrm{C}}=1: 4$, where $n_{\mathrm{C}}$ and $n_{\mathrm{O}}$ is the number of carbon and oxygen atoms (Pandey, Reifenberger \& Piner, 2008).

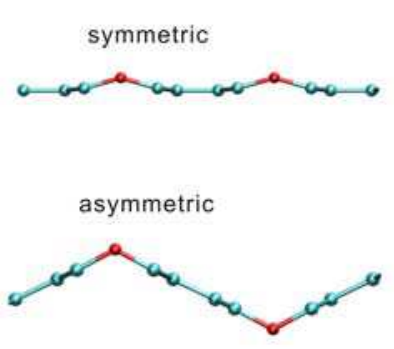

(a)

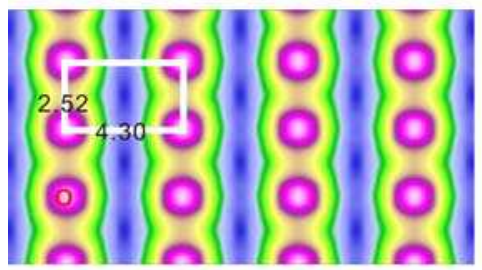

(c)

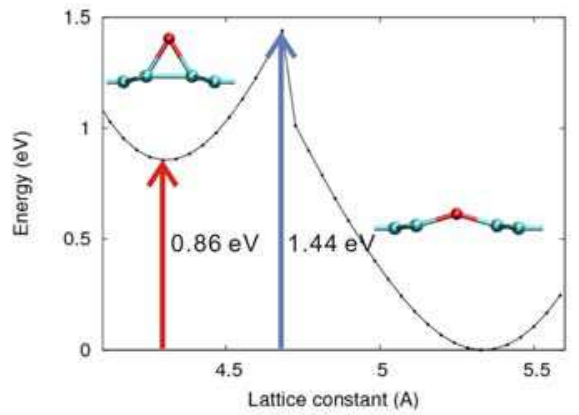

(b)

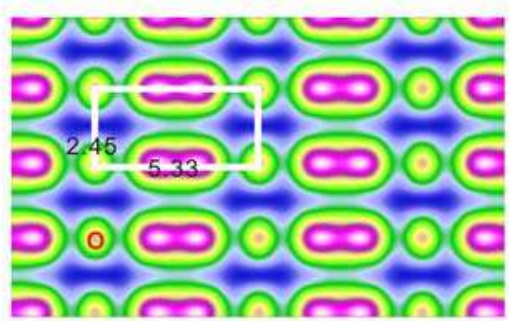

(d)

Fig. 6. (a) Optimized symmetric and antisymmetric structures for $\mathrm{C}_{8} \mathrm{O}$, projected along the epoxy line direction; (b) Energy landscape for graphene epoxide $\mathrm{C}_{4} \mathrm{O}$ as changing the lattice constant along the $x$ direction: insets show its metastable clamped (left) and unzipped (right) structures; (c) and (d) Simulated STM image for clamped (c) and unzipped (d) graphene oxide where epoxy groups bind on the same side. For the clamped phase, the simulated image and lattice constants $(\mathrm{a}=4.30 \AA$ and $\mathrm{b}=2.52 \AA)$ are consistent with experimental observations (Pandey, Reifenberger \& Piner, 2008; Xu \& Xue, 2010).

To clarify this inconsistence, DFT calculations are performed for graphene epoxide. The results show that in graphene epoxide $\mathrm{C}_{4} \mathrm{O}$, when oxygen atoms bind on the same side (symmetric structure in Fig. 6(a) and (b)), two distinct phases-metastable clamped and stable unzipped structures - are observed consistent with the two experimental observations (Fig. 6(b)). In the clamped structure, oxygen atoms form a regular lattice on the graphene sheet. This metastable structure is stabilized by an energy barrier of $0.58 \mathrm{eV}$. The simulated STM images (Fig. 6(c) and (d)) reproduce corresponding structures observed in the experiments. Furthermore, if both sides of the graphene are exposed for oxidation, neighboring epoxy groups can reside also on opposite sides to form the so-called antisymmetric structure. The formation energy of graphene epoxide in this symmetry is lower in unzipped phases (Fig. 6(b)). In the antisymmetric structures, the planar structure of the graphene part between epoxy groups is well kept and there is negligible lattice 
distortion in the graphene sheet. The final structure is optimized in a zigzag shape, where neighbouring epoxy groups hinge planar zigzag graphene ribbons.

\subsection{Coupling between graphene sheets and substrates}

Although suspended and freestanding graphene can be established by deposit graphene sheet on scaffolds or nano-pores, most applications require that graphene is supported by a substrate, which has a stable and planar morphology and is easy to pattern. Substrates with mismatched lattice constants and strong adhesion could induce ripples of several Angstroms in the graphene sheet. While those with similar lattice constants, e.g. Ni, preserve its planar geometry. The substrates where graphene sheets are deposited offer another route to modulate the ground state and transport properties of graphene and related materials (Witterlin \& Bocquet, 2009; Xu \& Buehler, 2010b).

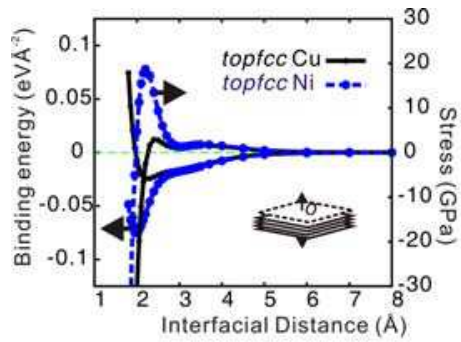

(a)

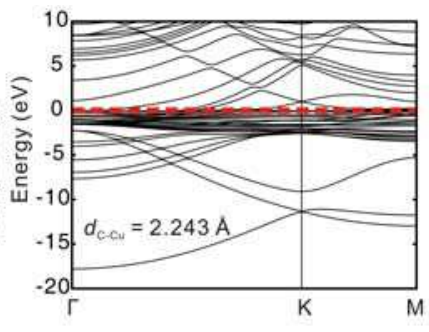

(b)

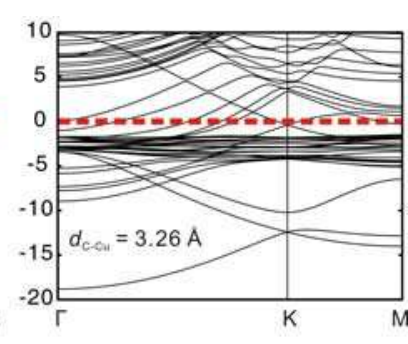

(c)

Fig. 7. (a) Interface binding energy and stress $\sigma$ between graphene and the copper/nickel (111) surfaces. The binding energy $E_{\mathrm{b}}$ is $-91.33 \mathrm{meV}^{-2}$ for nickel, higher than $-24.81 \mathrm{eV} \AA^{-2}$ for copper surface. Its tensile strength is $18.70 \mathrm{GPa}$, also much higher than $2.92 \mathrm{GPa}$ as obtained for the copper surface; (b) and (c) Electronic band structures of the graphenecopper (111) hybrid system with interface distance $d_{\mathrm{C}-\mathrm{Cu}}=2.243 \AA$ (b) and $3.26 \AA$ (c) (Xu \& Buehler, 2010b).

The binding between graphene and metal substrates is generally weaker than a covalent bond, and can be classified into two groups. $\mathrm{Al}, \mathrm{Cu}, \mathrm{Ag}$, $\mathrm{Au}$ and $\mathrm{Pt}$ have weak cohesion with graphene, while $\mathrm{Co}, \mathrm{Ni}$ and $\mathrm{Pd}$ have strong cohesion (Giovannetti et al., 2008). DFT calculations on copper (111) and nickel (111) surfaces adhered to a monolayer of graphene show that their cohesive energy, strength and electronic structure correlate directly with their atomistic geometry. Due to the strong coupling between $\Pi$-orbitals in graphene and open $d$-orbitals in metal, the nickel-graphene interface has much a stronger cohesive energy with graphene than copper (Fig. 7(a)). Interestingly, for both nickel and copper, the interface cohesive energy profile features a well-and-shoulder shape that cannot be captured by simple pair-wise models such as the Lennard-Jones potential. Band structure analysis shows that, the binding between graphene and metal substrate at the well and shoulder positions in the profile correspond to different electronic coupling at the interface. For example, at $d_{C}$ $\mathrm{Cu}=2.243 \AA$, the Fermi level $\left(E_{\mathrm{F}}=0\right)$ of the hybrid system is pinned to the $\mathrm{Cu}$ bands and $d_{\mathrm{C}}$ $\mathrm{Cu}$ is close to the interlayer distance in graphite. While at $d_{\mathrm{C}-\mathrm{Cu}}=3.26$, the coupling between the $p_{z}$ orbital of carbon atoms and the $d_{z 2}$ orbital of copper atoms is weak, the Fermi level is close to the crossing between graphene $\Pi$ and $\Pi^{*}$ bands, and $d_{\mathrm{C}-\mathrm{Cu}}$ is close to the interlayer distance in copper crystal along (111) direction (Xu \& Buehler, 2010b). These results provide a detailed understanding of the interfacial properties of graphene-metal systems, and help 
to predict the performance of graphene based nanoelectronics and nanocomposites. The availability of structural and energetic data of graphene-metal interfaces could also be useful for the development of empirical force fields for molecular dynamics simulations that correctly characterize their structural and mechanical properties.

Intercalation of atoms (metal, hydrogen, oxygen, nitrogen oxide, etc.) between graphene sheet and the substrate is another novel route to modify the interfacial chemistry. The selective oxidation of ruthenium surface beneath graphene can lift the strong Ru-graphene coupling and restores characteristic Dirac cones of monolayer graphene sheet (Sutter et al., 2010). Hydrogen intercalation can also decouple graphene sheet from silicon carbide surface that is initially covalently bonded and produce quasi-free-standing epitaxial graphene sheet on large SiC wafers (Riedl et al., 2009).

\subsection{Interior doping through defects}

Creating defects, localized or extended, can also modify graphene and form nanostructures interiorly (Lusk \& Carr, 2008; Bai et al., 2010), in comparison to the exterior doping as discussed previously. Grain boundaries introduced by extended local defects, such as StoneWales defects and divacancies, can alter the electronic structures of graphene sheet (Yazyev \& Louie, 2010). A one-dimensional topological defect containing octagonal and pentagonal $s p^{2}$-hybridized carbon rings embedded in a perfect graphene sheet act as a quasi-onedimensional metallic wire (Lahiri et al., 2010).

\subsection{Effects of external fields}

It is reported that the bandgap of bilayer graphene can be tuned by electric field in perpendicular to the basal plane, e.g. through gating. The symmetry of sublattice in $A B$ stacking is broken upon electrical gating. The electrical displacement fields in different graphene layers produce two effects including a net carrier doping (shifting of the Fermi energy) and generation of a non-zero bandgap. The gate-tunable bandgap can reach 250 $\mathrm{meV}$ and has the unusually strong oscillator strength for the bandgap transitions. Thus bilayer graphene under electrical field tuning can enable novel nanophotonic devices for infrared light generation, amplification and detection. Using a double-gate configuration, an insulating state with large suppression of the conductivity in bilayer graphene can be further achieved (Zhang et al., 2009; Oostinga et al., 2008). In comparison to exterior dopants and defects, the advantage of this control method is that the control is non-contact and can be continuous tuned.

\section{Strain engineering}

Strain engineering, or more generally tuning material properties by applying mechanical loads or deformation, is a powerful strategy in improving material performance. In siliconbased semiconductor industry, strained silicon has much higher mobility, and thus much better chip performance and lower energy consumption. When material goes down to low dimension, the effects of strain become even more significant as directional load is more feasible. Also bending of two-dimensional membranes or one-dimensional ribbons can easily be excited at ambient condition, resulting in a significant local curvature. Recently, stretchable and flexible electronics have attracted many interests from various disciplines. Thus, a mapping between deformation of graphene and its impacts on the properties becomes extremely important. 
As introduced in previous sections, molecular functionalization on graphene sheet usually induces structural distortion as it disturbs or breaks the underlying $s p^{2}$ bonding network and forms $s p^{3}$ bonds locally. Vice versa, mechanical deformation, which modifies the $s p^{2}$ bonds, provides another method to tune the graphene properties, reversibly. This approach, in combination with molecular doping, is expected to play a critical role in nano-engineering of graphene and related materials.

\subsection{Structural and structural response to molecular doping}

When graphene is hydrogenized by single hydrogen atom, the dangling $p_{z}$ state of carbon is saturized by the proton. As a result the $s p^{2}$ network below is broken. Distortion is formed, tending to transform the planar morphology into tetrahedral lattice as in diamond. The fully hydrogenated graphane structure, as shown in Fig. 8, can take two forms with hydrogen binding on the same or alternating sides of A and B lattices. We call them symmetrical and anti-symmetrical graphane, respectively.

In comparison to pristine graphene where $a_{\mathrm{g}}=2.44 \AA$, consistent with experimental value $2.42 \AA$, these two graphane phases have lattice constants $a_{\mathrm{sym}}=2.79 \AA$ and $a_{\mathrm{asym}}=2.5 \AA$, respectively. The antisymmetric phase is found to be energetically $1.8 \mathrm{eV}$ (per carbonhydrogen pair) more favorable than the symmetric one.

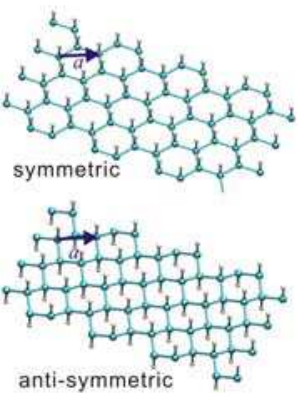

(a)

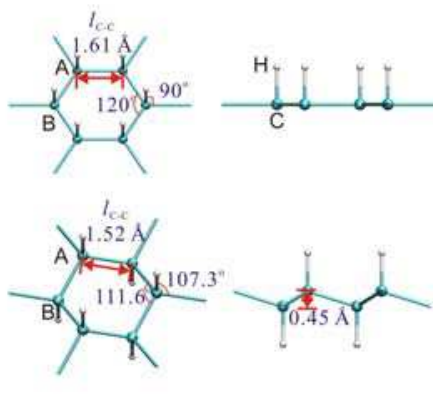

(b)

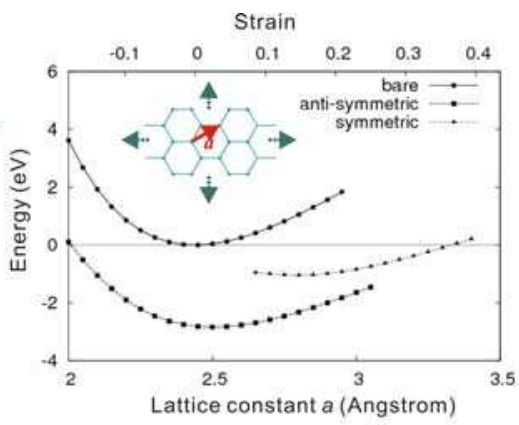

(c)

Fig. 8. (a) Optimized structures of symmetric and antisymmetric graphane. The unit cell contains two carbon atoms (A and B denote the two sublattices) as in graphene and two hydrogen atoms; (b) Local atomic structures; (c) Energies of pristine graphene (with additional energies from isolated hydrogen atoms) and graphane under biaxial strain loading showing the effects of strain engineering (Xue \& Xu, 2010a).

In the symmetric phase (Fig. 8 a and b), binding of hydrogen atoms expands the lattice constant underneath hexagonal graphene lattice by $14 \%$, while preserving the planar configuration. As evidenced by localized density of states close to the Fermi level, the planar symmetry preserves $s p^{2}$ characteristic in graphane. Total density of states is projected onto $s$ and $p$ atomic orbital on each carbon atoms. $p_{\mathrm{z}}(l=1, m=0)$ orbital is distinct and contributes mostly near the Fermi level. Binding of hydrogen induces 0.14 electron transfer from hydrogen to carbon since carbon is slightly more electronegative than hydrogen. Also the hydrogenation through $p_{\mathrm{z}}$ orbital opens an energy gap $E_{\mathrm{g}}$ of $0.26 \mathrm{eV}$ in comparison with the zero-gap nature of pristine graphene.

In antisymmetric phase (Fig. 8 a and b), symmetry breaking between the two representative carbon (hydrogen) atoms in a unit cell allows the transition from $s p^{2}$ (graphene-like) 
hybridization to $s p^{3}$ (diamond-like) characteristics. The $s p^{3}$ hybridization induces out-ofplane corrugation of $0.45 \AA$ and the carbon-carbon bond length is $1.52 \AA$, which is close to $1.54 \AA$ in diamond. The bond angle $A_{\mathrm{C}-\mathrm{C}-\mathrm{C}}=111.6^{\circ}$ and $A_{\mathrm{H}-\mathrm{C}-\mathrm{C}}=107.3^{\circ}$ also resemble the tetrahedral angle $109^{\circ}$ in diamond. The antisymmetric phase also opens a bandgap $E_{\mathrm{g}}$ of 3.35 $\mathrm{eV}$, which is smaller than that in diamond (4.2 eV from DFT calculation).

In the symmetric structure, all the bond distortion has the same sign of curvature, thus forcing the sheet to bend towards the hydrogen-free side. This mechanism is proposed to form nanoscrolls or nanotubes by hydrogenating suspended graphene sheet $(\mathrm{Yu} \& \mathrm{Liu}$, 2007). However, in the anti-symmetric graphane, although the structure is corrugated due to the torsion of carbon-carbon bonds, the whole structure keeps two-dimensional.

Oxidation by epoxy groups also induces significant change of the graphene structure as discussed in the previous section. For the symmetrical structure, the epoxy ring is close to an equilateral triangle in the metastable clamped phase. The adsorption of an oxygen atom stretches the lower $\mathrm{C}-\mathrm{C}$ bonds by $5 \%$ but does not break the $s p^{2}$ carbon network (Fig. $6(\mathrm{~b})$ ). In the more stable unzipped structure, the angle $\mathrm{C}-\mathrm{O}-\mathrm{C}$ between two carbon-oxygen bonds in an unzipped epoxy ring is about $150^{\circ}$. The final $\mathrm{C}-\mathrm{C}$ bond is broken with an extended distance of $2.5 \AA$ (Fig. 6(a and b)), explaining the folds and wrinkles observed in graphene oxides due to sparse epoxy lines (Li et al., 2006).

In the unzipped phase, an epoxy group breaks the lower $s p^{2}$ bond and modifies the mechanical and electronic properties of graphene remarkably. The foldable epoxy ring structure reduces its Young's modulus by $42.4 \%$, while leaving the tensile strength almost unchanged. As $n_{\mathrm{O}}: n_{\mathrm{C}}$ is decreasing, the perturbation of epoxidation on the band structures depends on the density and symmetry of oxidation. These oxygenated monolayer sheets are structurally close to graphene and are expected to have similarly peculiar properties. One thus can functionalize graphene structures in a controllable manner through chemical oxidation and reduction (Xu \& Xue, 2010).

\subsection{Effects of structural deformation on the properties of graphene materials}

Elastic strain can induce a shift of the Dirac point energy from local changes in electronic density. Moreover, it will also induce an effective vector due to the change of electronhoppling amplitude between adjacent carbon atoms (Castro Neto et al., 2009). Local buckling, wrinkling and curving of graphene sheet also generate pseudoelectric and pseudomagnetic fields that modify its electronic structures and transport properties (Pereira et al., 2010; Bao et al., 2009). The charge carriers in graphene are expected to circulate as if under the influence of an applied out-of-plane magnetic field. It has recently been proposed that a modest strain field with triangular symmetry will give approximately uniform, quantizing magnetic fields upward of tens of Tesla (Levy et al., 2010).

The abrupt change in lattice constants and planar structure of graphene after hydrogenation in symmetric and antisymmetric phase suggests possible control of the hydrogenation process and the properties of graphane through applying mechanical deformation. Strain engineering is proposed to tune the hydrogenation process on graphene by applying deformation to graphene in prior to hydrogen binding or releasing. To quantify the binding strength between the hydrogen atoms and graphene sheet in terms of single carbonhydrogen pair, the formation energy of graphane is defined as $E_{\mathrm{f}}=E_{\text {graphane }}-\left(E_{\text {graphene }}+E_{\mathrm{H}}\right)$, where $E_{\text {graphane }}$ and $E_{\text {graphene }}$ are the energy of graphane and pristine graphene, respectively. In absence of strain in the graphene lattice, symmetric and antisymmetric graphane phases (Fig. 8(a) and (b)) have binding strength $E_{\mathrm{f}}$ of -1.044 and $-2.847 \mathrm{eV}$ per $\mathrm{C}-\mathrm{H}$ atom pair. 
Fig. 8(c) show the effects of biaxial in-plane strain on the energies of both the types of graphane structures, and also the combined system of pristine graphene and isolated hydrogen atom. Pristine graphene has an optimized lattice constant $a_{\mathrm{g}}=2.44 \AA$, while symmetric graphane has $a_{\text {sym }}=2.79 \AA$. The energy of both graphene (with additional constant energy terms from isolated hydrogen atoms) and symmetric graphane increases as the tensile strain is enhanced, When the lattice constant a exceeds $3.35 \AA$, the binding energy of symmetric graphane is larger than the value of undeformed system with pristine graphene and isolated hydrogen atoms. Also energy of nonhydrogenated system increases much faster than symmetric graphane. As a result, the binding energy of hydrogen is changed by $53.89 \%$ (with respect to the value without loading, a $=2.44 \AA$ ) when the graphene is under a prestrain of $10 \%$ (from a $=2.44$ to $2.69 \AA$ ). The strain for $s p^{2}$ bond breaking in graphene is $18 \%$, enabling a feasibly reversible control. On the other hand, if the graphene sheet is restrained at $a_{\mathrm{g}}=2.44 \AA$ against to in-plane expansion or the symmetric graphane is compressed from its equilibrium value to below $2.65 \AA$, repulsion between the carbon-hydrogen pairs will cause the hydrogenated structure to be mechanically unstable. The hydrogen atoms are repelled (released) from the graphene plane. The sensitivity of symmetric hydrogenation of graphene on strain observed here thus provides a flexible and reversible controllability by simply stretching or compressing the graphene sheet.

In antisymmetric phase, a remarkable dependence of the formation energy on the strain is also observed. Optimized lattice constant $a_{\text {asym }}=2.5 \AA$. In-plane tensile and compressive strain of graphene sheet up to $10 \%$ can change the binding strength remarkably by $23.56 \%$ and $-2.9 \%$. The structure breaks at a tensile strain of $22 \%$ or a compressive strain of $26 \%$. In comparison with symmetric phase, the strain modification of hydrogen binding energy is less significant as it has similar optimal lattice constant as pristine graphene. Under tensile strain, due to the weaker interaction between adjacent carbon-hydrogen pairs on the opposite sides of graphene, the expansion of in-plane lattice constant is found to be able to reduce its out-of-plane corrugation slightly and partially recover the $\Pi$ electron characteristics of graphene.

In symmetric phase, carbon-hydrogen bond length $l_{\mathrm{C}-\mathrm{H}}$ increases at either tensile or compressive strain. As $a$ is reduced to be less than $2.6 \AA$, the carbonhydrogen bond is broken. Hydrogen atoms on graphene will be released from graphene sheet as discussed before. While at tensile strain and when the stress approaches the tensile strength and the in-plane $s p^{2}$ carbon network is about to be broken, $l_{\mathrm{C}-\mathrm{H}}$ is elongated to the same value as in benzene or methane $\left(l_{\mathrm{CH} \text {-methane }}=1.096 \AA\right.$ ). In antisymmetric phase, when tensile strain is applied, the planar configuration of graphene is partially recovered, suggesting emerging $s p^{2}$ feature. The carbon-hydrogen bond length decreases and approaches the value in $s p^{2}$ bonded symmetric phase in this situation. When antisymmetric structure is compressed, the $s p^{3}$ characteristic is enhanced and the carbon-hydrogen bond length also decreases toward the value in methane. As a result, the carbon-hydrogen bond length maximizes at the optimised lattice constant. These remarkable strain effects can further be extended to other nanostructures like carbon nanotubes and few-layer graphite (Xue \& Xu, 2010a; Xue \& Xu, 2010b).

\section{Hierarchical structures of macroscopic graphene materials}

Graphene and graphene nanoribbons present intriguing properties and thus have been suggested for a wide range of applications from nanoelectronics to nanoelectromechanical 
systems. However, as introduced before (Fig. 1), the morphology of this monolayer material has large derivation from the native two-dimensional sheet at elevated structures, especially for the ribbon with high aspect ratio. Also entanglements and scrolls are easy to form at marked density, instead of the patterned or self-organized structures as required by industrial applications. To bridge the scales from their nanostructural geometry - the key for their unique properties - to the critical requirements for large-scale electronics and device applications, there we need a hierarchical approach to assembly macroscopic materials from functionalized graphene sheets or nanoribbons. This approach has to (1) be able to assemble the nanostructures into macroscopic assemblies, while (2) preserving their nanostructurespecific physical significance and novel properties at larger scales, such as its size- and shape-dependent electronic structures.

\subsection{Hierarchical assembly of functionalized graphene nanostructures}

As inspired by the hierarchical bonding networks in protein and nucleic acid materials (Fig. 9(a)), a bottom-up assembly or top-down lithography approach has been proposed by introducing hydrogen bonds between individual graphene nanoribbons (Fig. 2(b), Xu \& Buehler, 2009). After substitutional doping by oxygen and nitrogen atoms on carbon atoms at edges of graphene ribbons, hydrogen bonds $(-\mathrm{C}=\mathrm{O} \ldots \mathrm{H}-\mathrm{N}-$ or $-\mathrm{N} \ldots \mathrm{H}-\mathrm{N}-)$ are formed between each GNR monomer. DFT calculations show that a two-dimensional sheet consisting of GNR arrays can be stabilized through these directional weak bonds, mimicking the structure of beta-sheet proteins or base-pairs connecting DNA or RNA strands (Fig. $9(\mathrm{~b}))$. The cohesive energy for each $-\mathrm{C}=\mathrm{O} \ldots \mathrm{H}-\mathrm{N}$ - hydrogen bond is $29.98 \mathrm{kcalmol}^{-1}$, which is considerably higher than the typical value $2.8 \mathrm{kcalmol}^{-1}$ in organics. This binding energy level resides in the realm of 'strong hydrogen bond' nature and reflects the cooperativity of periodic hydrogen bonds arrangements. The hierarchical structure is mechanically stablized by a tensile strength of $1.08 \mathrm{GPa}$ in parallel to the hydrogen bond direction and a shear strength of $1.78 \mathrm{GPa}$ across the hydrogen bond network.

Remarkably, these assemblies, as predicted by both first-principles calculations and molecular dynamics simulations, not only are energetically and mechanically stable, but also preserve the unique electronic properties of individual graphene nanoribbons in the bulk. Specifically, the energy gap of the bulk material shrinks as the width of the constituting graphene nanoribbon increases. For instance, the bandgap $E_{\mathrm{g}}$ and $\Pi-\Pi^{*}$ energy gap for $-\mathrm{C}=\mathrm{O} \ldots \mathrm{H}-\mathrm{N}$ - assembly decreases from 1.5 and $0.5 \mathrm{eV}$ at $w=1 \mathrm{~nm}$ to zero at 3.6 and $2.8 \mathrm{~nm}$ respectively (Fig. 9(c)). This tunability of bulk material properties through controlling the nanostructure enables the synthesis of a broader class of biomimetic multifunctional mechanomutable and electromutable nanomaterials for electromechanical applications. It's also notable that the localized density of states of valence and conduction bands locates at different spatial region in the assembly (Fig. 9(d)). Effective charge separation could thus be achieved by forming such junctions for photovoltaic applications.

\subsection{Graphene based nanocomposites}

Another class of macroscopic assemblies from graphene sheets are nanocomposites. Carbon materials, as special types of polymers, have been widely used as the filler phases to enhance the mechanical, thermal and electrical properties of polymers or metals. The carbon fiber is the most notable example of this material class, which has been developed and widely used from the 1960s and is still spreading in many industries today. 


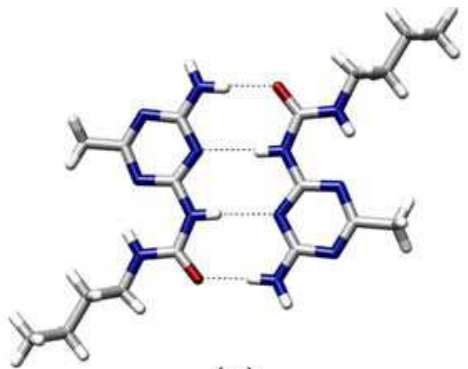

(a)

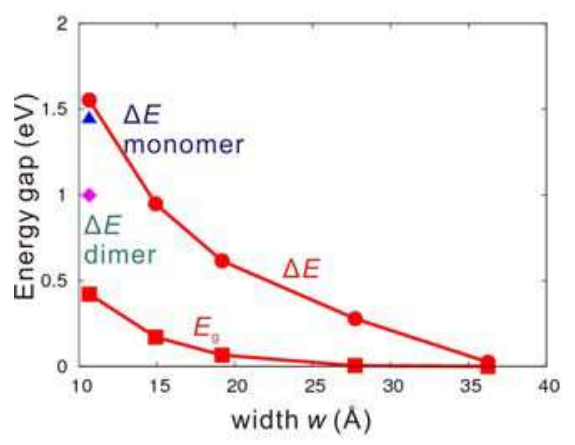

(c)

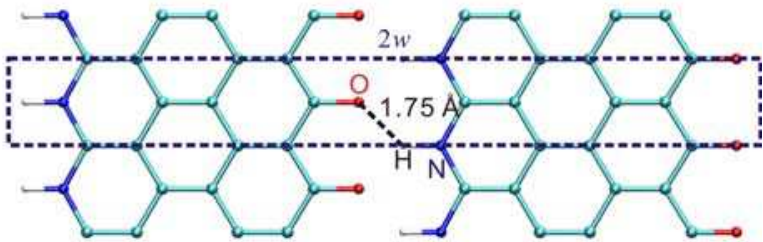

(b)

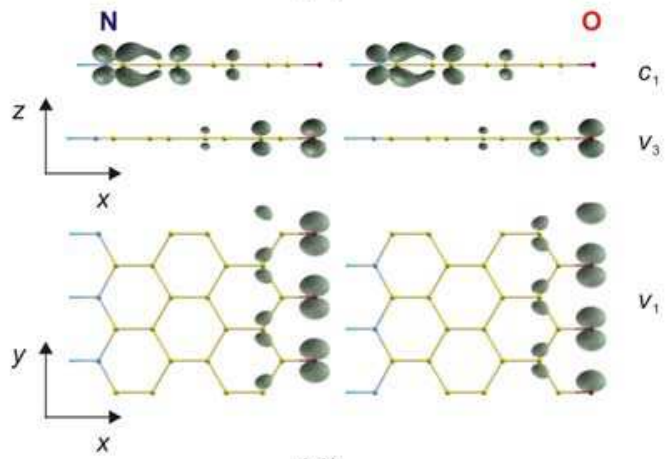

(d)

Fig. 9. (a) Hydrogen bond network in proteins; (b) Atomic structure of the assemblies, resembling the geometry of beta-sheet proteins. The dashed box shows a unit cell with width $2 w$, where $w$ is the width of individual GNR; (c) Energy gap $E_{\mathrm{g}}$ (circle line) and $\Pi-\Pi^{*}$ bandgap $\Delta E=E_{\Pi^{*}}-E_{\Pi}$ of monomer, dimer and hierarchical graphene nanoribbon assemblies through $-\mathrm{C}=\mathrm{O}$...H-N- hydrogen bonds (square line) with different widths; $(\mathrm{d})$ Local densities of states for $\Pi^{*}\left(c_{1}\right), \Pi\left(v_{3}\right)$ and oxygen $\left(v_{1}\right)$ states, which are all localized at the graphene edge and decay quickly into the ribbon (Xu \& Buehler, 2009).

The key issues determining performance of these composites are to (1) effectively disperse the filler phase and (2) improve the load or energy transfer between filler and matrix. Graphene sheets have highly appreciated structural and thermal stability, outstanding mechanical and thermal, electrical transport properties. However, the cohesion between graphene sheets in graphite platelets and weak van der Waal binding between graphene and polymer matrix greatly prohibit the load transfer, distribution, and thus its application in high-performance nanocomposites.

However, recent progresses in functionalized graphene, such as graphene oxide nanocomposites, successfully solve these two critical issues. Firstly, the formation of epoxy, hydroxyl and other functional groups on the basal plane of graphene sheet expands the interlayer distances in graphite and avoids multiplayer aggregation. Secondly, through forming hydrogen bonds, metal-mediated bonds or polymerisation between the graphene oxide sheets and polymers, the performance can be effectively improved, thus providing in parallel excellent performance and cheap fabrication.

In comparison to the buckypapers consisting of carbon nanotubes and other carbon or claybased papers, Ruoff's group find that the graphene oxide paper-like material possesses 
unique layered structures where individual compliant graphene oxide sheets are interlocked/tiled together in a near-parallel fashion. Their atomic-scale corrugation, microscale wrinkled morphology and strong interlayer interactions results in a highly effected load distribution across the entire macroscopic sample and thus make the material more resilient than other papers (Dikin et al., 2007). Impressively, they find that the graphene oxides are feasible for further functionalization. Significant enhancements in mechanical stiffness $(10-200 \%)$ and fracture strength $(\sim 50 \%)$ are achieved by introducing a small amount (less than $1 \mathrm{wt} \%$ ) of divalent ions, e.g. $\mathrm{Mg}^{2+}$ and $\mathrm{Ca}^{2+}$ (Park et al., 2008). Chemical modification with alkylamines can also be established that directly introduces tunability of paper structures and tensile strength through the amine length (Stankovich et al., 2010).

\section{Conclusion and perspectives}

In this chapter, we outline recent research progresses on nano-engineering the graphene and related materials. The content is classified into four categories: structural tailoring, molecular doping, strain engineering and hierarchical assembling. Each of these categories is elaborated with both the general idea, related theoretical and experimental work. The topics selected here are those the author has been working on, to ensure we include as much exciting achievement as possible in this fertilized field.

Nevertheless, there are still several key issues expected to be resolved in the future development on the graphene nano-engineering:

Scalability - Most of the nano-engineering techniques modify the properties of graphene and related materials at the molecular level. However towards industrial applications, feasible scaling methods have to be developed, such as patterning and self-assembly. Unlike other self-assembly system, the scaling here on graphene sheet must be hierarchical, the layer-bylayer or scrolling organizing have to be exclude in the assembling process as they introduce interlayer coupling between graphene sheets. The controllable top-down lithography and bottom-up assembly directed by hydrogen bonds or covalent bonds that have orientationspecification are very promising in this direction.

Tunability - Multifunctional materials hold great potentials for the next-generation materials including high-performance composites, nanoelectronics, microfluidics, and other microelectromechanical systems (MEMS) or nanoelectromechanical systems (NEMS). The non-covalent functionalization methods treating gaseous molecules, metal atoms, or covalent functionalization that can be reversibly applied have great potentials for this issue. Applications - The multifunctionality of graphene offers it numerous applications. Novel applications in emerging fields still need exploration, such as quantum information devices and sustainable energy applications where nanoscale engineering can play an important role. Moreover, the graphene flake, nanoribbon, sheet and graphite are representative zero-, one-, two- and three-dimensional materials. This unique feature suggests to use graphene related materials as platforms or model materials for basic research. Graphene is also a unique material standing between inorganic and organics materials. Combining materials engineering with synthetic biological methods also helps to select, design and produce new classes of materials designed from the molecular level upwards.

Finally, we close this chapter by calling upon researchers to pay attention to following key scientific problems.

Intermolecular interactions - The basic interactions between molecular dopants, functional groups, supporting substrates and graphene are still not clear. The physical and chemical 
properties of interactions such as hydrogen bonds, electrostatic, hydrophobic forces, especially those involved in assembling processes, should be highlighted.

Nanoscale interfaces - The interface between graphene and various materials including semiconductors, metals and polymers dominates the performance of nanoelectromechanical devices and nanocomposites. However, it is not clear that if conventional interface theories still hold at this scale, especially those related to the energy transport processes across.

Multiphysics coupling - The research on graphene and related materials towards industrial applications are intrinsically multiphysical and multiscale. The interplay between structural, mechanical, electronic and optical properties, which shapes the unique features of these materials outperforming conventional materials, must be understood. Multiphysics and multiscale computational paradigm and experimental platforms are urged.

\section{References}

Bae, S. et al. (2010). Roll-to-roll production of 30-inch graphene films for transparent electrodes, Nature Nanotechnology 5, 8, 574-578

Bai, J. et al. (2010). Graphene nanomesh, Nature Nanotechnology 5, 3, 190-194

Bao, W. et al. (2009). Controlled ripple texturing of suspended graphene and ultrathin graphite membranes, Nature Nanotechnology 4, 9, 562-256

Bartels, L. (2010). Tailoring molecular layers at metal surfaces, Nature Chemistry 2, 2, 87-95.

Bets, K. and B. Yakobson (2009). Spontaneous twist and intrinsic instabilities of pristine graphene nanoribbons, Nano Research 2, 2, 161-166

Cai, J. et al. (2010). Atomically precise bottom-up fabrication of graphene nanoribbons, Nature 466, 7305, 470-473

Castro Neto, A. H. et al. (2009). The electronic properties of graphne, Review of Modern Physics 81, 1, 109-162

Chen, Z., et al. (2007). Graphene nano-ribbon electronics, Physica E: Low-dimensional Systems and Nanostructures, 40, 2, 228-232

Ci, L. et al. (2008). Nanocutting of graphene, Nano Reseach 1, 2, 116-122

Dikin, D. A. et al. (2007). Preparation and characterization of graphene oxide paper, Nature $448,7152,457-460$

Echtermeyer, T. J. et al. (2007). A graphene-based electrochemical switch, Arxiv :0712.2026

Elias, D. C. et al. (2009). Control of graphene's properties by reversible hydrogenation: evidence for graphane, Science 323, 5914, 610-613

Ezawa, M. (2006). Peculiar width dependence of the electronic properties of carbon nanoribbons. Physical Review B 73, 4, 045432.

Ezawa, M. (2009). Spin filter, spin amplifier and spin diode in graphene nanodisk, The European Physical Journal B 67, 4, 543-549

Fasolino, A., Los, J. H. \& Katsnelson, M. I. (2007). Intrinsic ripples in graphene, Nature Materials 6, 6, 858-861

Fujii, S. \& Enoki, T. (2010). Cutting of oxided graphene into nanosized pieces, Journal of the American Chemical Society 132, 29, 10034-10042.

Fujita, M. et al. (1996). Peculiar localized state at zigzag graphite edge, Journal of the Physical Society of Japan 65, 7, 1920-1923

Giovannetti, G. et al. (2008) Doping graphene with metal contacts, Physical Review Letters $101,2,026803-4$ 
Girit, Ç. Ö. et al. (2009). Graphene at the edge: stabilityand dynamics, Science 323, 5922, 17021708

Guinea, F. et al. (2010). Energy gaps and a zero-field quantum Hall effect in graphene by strain enginering, Nature Physics 6, 1, 30-33

Gunlycke, D. et al. (2010). Edges bring new dimension to graphene nanoribbons, Nano Letters 10, 9, 3638-3642

Han, M. Y. et al. (2007). Energy bandgap engineering of graphene nanoribbons, Physical Review Letters 98, 20, 206805-4

Jia, X. et al. (2009). Controlled formation of sharp zigzag and armchair edges in graphitic nanoribbons, Science 323, 5922, 1702-1705

Jiao, L. et al. (2009). Narrow graphene nanoribbons from carbon nanotubes, Nature 458, 7240, $877-880$

Kawai, T. et al. (2009). Self-redirection of tearing edges in graphene: tight-binding molecular dynamics simulations, Physical Review B 80, 3, 033401-4

Kosynkin, D. V. et al. (2009). Longitudinal unzipping of carbon nanotubs to form graphene nanoribbons, Nature 458, 7240, 872-876

Lahiri, J. et al (2010). An extended defect in graphene as a metallic wire, Nature Nanotechnology 5, 5, 326-329

Lee, J.-H. \& Grossman, J. C. (2010). Magnetic properties in graphene-graphane superlattices, Applied Physics Letters 97, 13, 133102-3

Lerf, A. et al. (1998). Structure of graphite oxide revisited, Journal of Physical Chemistry B 102, $23,4477-4482$

Levy, N. et al. (2010). Strain-induced pseudo-magnetic fields greater than 300 tesla in graphene nanobubbles, Science 329, 5991, 544-547

Li, J.-L. et al. (2006). Oxygen-driven unzipping of graphitic materials, Physical Review Letters $96,17,176101-4$

$\mathrm{Li}$, X. et al. (2008). Chemically derived, ultrasmooth graphene nanoribbon semiconductors, Science 319, 5867, 1229-1232

Lusk, M. T. \& Carr, L. D. (2008). Nanoengineering defect structures on graphene, Physical Review Letters 100, 17, 175502-4

Meyer, J. C. et al. (2007). The structure of suspended graphene membrane, Nature 446, 7131, 60-63

Nakada, K. et al. (1996). Edge state in graphene ribbons: nanometer size effect and edge shape dependence, Physical Review B 54, 24, 17954-17961

Novoselov, K. S. et al. (2004). Electric field effect in atomically thin carbon films, Science 306, $5696,666-669$

Oostinga, J. B. et al. (2008). Gate-induced insulating state in bilayer graphene devices, Nature Materials 7, 2, 151-157

Pandey, D., Reifenberger, R. \& Piner, R. (2008). Scanning probe microscopy study of exfoliated oxidized graphene sheets, Surface Science 602, 9, 1607-13

Park, S. et al. (2008). Graphene oxide papers modified by divalent ions - enhancing mechanical properties via chemical cross-linksing, ACS Nano 2, 3, 572-578

Pereira, V. M. et al. (2010). Geometry, Mechanics, and Electronics of Singular Structures and Wrinkles in Graphene, Physical Review Letters 105, 15, 156603-4

Riedl, C. et al. (2009). Quasi-free-standing epitaxial graphene on SiC obtained by hydrogen intercalation, Physical Review Letters 103, 24, 246804. 
Schedin, F. et al. (2007). Detection of individual gas molecules adsorbed on graphene, Nature Materials 6, 9, 652-655

Sen, D. et al. (2010). Tearing graphene sheets from adhesive substrates produces tapered nanoribbons, Small 6, 10, 1108-1116

Shi, X. et al. (2010). Tunable water channels with carbon nanoscrolls, Small 6, 6, 739-744

Singh, A. \& Yakobson, B. I. (2009). Electronics and magnetism of patterned graphene nanoroads, Nano Letters 9, 4, 1540-1543

Son, Y. W., Cohen, M. L. \& Louie, S. G. (2006a). Half-metallic graphene nanoribbons, Nature $444,7117,347-349$

Son, Y. W., Cohen, M. L. \& Louie, S. G. (2006b). Energy gaps in graphene nanoribbons, Physical Review Letters 97, 21, 216803-4

Stankovich, S. et al. (2010). Systematic Post-assembly modification of graphene oxide paper with primary alkylamines, Chemistry of Materials 22, 14, 4153-4157

Sutter, P., Sadowski, J. T. \& Sutter, E. A. (2010). Chemistry under cover: tuning metalgraphene interaction by reactive intercalation, Journal of the American Chemical Society 132, 23, 8175-8179

Tapasztó, L. et al. (2008). Tailoring the atomic structure of graphene nanoribbons by scanning tunneling microscope lithography, Nature Nanotechnology 3, 7, 397-401.

Wang, W. L. et al. (2007). Graphene nanoflakes with large spin, Nano Letters 8, 1, 241-245

Wintterlin, J. \& Bocquet, M. L. (2009). Graphene on metal surfaces, Surface Science 603, 10-12, $1841-1852$

Xie, X. et al. (2009). Controlled Fabrication of high-quality carbon nanoscrolls from monolayer graphene, Nano Letters 9, 7, 2565-2570

$\mathrm{Xu}, \mathrm{Z}$.; Zheng, Q. \& Chen, G. (2007). Elementary building blocks of graphene-nanoribbonbased electronic devices, Applied Physics Letters 90, 22, 223115-3

Xu, Z. \& Buehler, M. J. (2009). Hierarchical graphene nanoribbon assemblies feature unique electronic and mechanical properties, Nanotechnology 20, 37, 375704-8

$\mathrm{Xu}, \mathrm{Z}$. (2009). Graphene nanoribbon under tension, Journal of Computational and Theoretical Nanoscience 6, 3, 625-628

Xu, Z. \& Xue, K. (2010). Engineering graphene by oxidation: a first principles study, Nanotechnology 21, 4, 045704-7

Xu, Z. \& Buehler, M. J. (2010a). Geometry controls conformation of graphene sheets: Membranes, ribbons and scrolls, ACS Nano 4, 7, 3869-3876

$\mathrm{Xu}, \mathrm{Z}$. \& Buehler, M. J. (2010b). Interface structure and mechanics between graphene and metal substrates, Journal of Physics : Condensed Matter 22, 48, 485301-5

Xue, K. \& Xu, Z. (2010a). Strain effects on basal-plane hydrogenation of graphene: a firstprinciples study, Applied Physics Letters 96, 6, 063103-3

Xue, K. \& Xu, Z. (2010b). Hydrogenation of carbon nanotubes: roles of symmetry and strain, Journal of Computational and Theoretical Nanoscience (in the press)

Yang, X. et al. (2008). Two-dimensional graphene nanoribbons, Journal of the American Chemical Society, 130, 13, 4216-4217.

Yazyev, O. V. and S. G. Louie (2010). Electronic transport in polycrystalline graphene, Nature Materials 9, 10, 806-809

$\mathrm{Yu}, \mathrm{D}$. \& Liu, F. (2007). Synthesis of carbon nanotubes by rolling up patterned graphene nanoribbons using selective atomic adsorption, Nano Letters 7, 10, 3046-3050 
Zhang, Y.-H. et al. (2010). Tuning the electronic structure and transport properties of graphene by noncovalent functionalization: effects of organic donor, acceptor and metal atoms, Nanotechnology 21, 6, 065201-7

Zhang, Y. et al. (2009). Direct observation of a widely tunable bandgap in bilayer graphene, Nature 459, 7248, 820-823. 


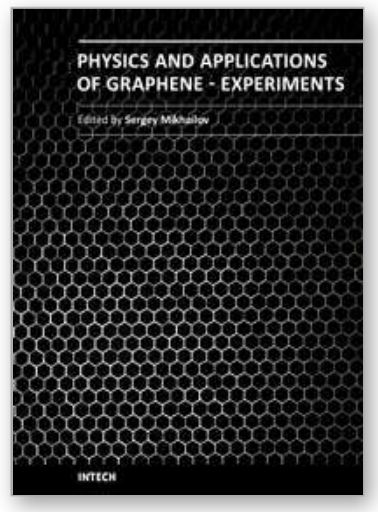

\author{
Physics and Applications of Graphene - Experiments \\ Edited by Dr. Sergey Mikhailov
}

ISBN 978-953-307-217-3

Hard cover, 540 pages

Publisher InTech

Published online 19, April, 2011

Published in print edition April, 2011

The Stone Age, the Bronze Age, the Iron Age... Every global epoch in the history of the mankind is characterized by materials used in it. In 2004 a new era in material science was opened: the era of graphene or, more generally, of two-dimensional materials. Graphene is the strongest and the most stretchable known material, it has the record thermal conductivity and the very high mobility of charge carriers. It demonstrates many interesting fundamental physical effects and promises a lot of applications, among which are conductive ink, terahertz transistors, ultrafast photodetectors and bendable touch screens. In 2010 Andre Geim and Konstantin Novoselov were awarded the Nobel Prize in Physics "for groundbreaking experiments regarding the two-dimensional material graphene". The two volumes Physics and Applications of Graphene - Experiments and Physics and Applications of Graphene - Theory contain a collection of research articles reporting on different aspects of experimental and theoretical studies of this new material.

\title{
How to reference
}

In order to correctly reference this scholarly work, feel free to copy and paste the following:

Zhiping Xu (2011). Nano-Engineering of Graphene and Related Materials, Physics and Applications of Graphene - Experiments, Dr. Sergey Mikhailov (Ed.), ISBN: 978-953-307-217-3, InTech, Available from: http://www.intechopen.com/books/physics-and-applications-of-graphene-experiments/nano-engineering-ofgraphene-and-related-materials

\section{INTECH}

open science | open minds

\section{InTech Europe}

University Campus STeP Ri

Slavka Krautzeka 83/A

51000 Rijeka, Croatia

Phone: +385 (51) 770447

Fax: +385 (51) 686166

www.intechopen.com

\section{InTech China}

Unit 405, Office Block, Hotel Equatorial Shanghai

No.65, Yan An Road (West), Shanghai, 200040, China

中国上海市延安西路65号上海国际贵都大饭店办公楼 405 单元

Phone: +86-21-62489820

Fax: $+86-21-62489821$ 
(C) 2011 The Author(s). Licensee IntechOpen. This chapter is distributed under the terms of the Creative Commons Attribution-NonCommercialShareAlike-3.0 License, which permits use, distribution and reproduction for non-commercial purposes, provided the original is properly cited and derivative works building on this content are distributed under the same license. 